\title{
Calculation of Percentage of Coarse Aggregate Present in Concrete Using Processing of Digital Images Obtained with a Commercial Scanner
}

\author{
Jose Renato de Castro Pessoa ${ }^{1,2 *}$, Joel Sánchez Domínguez ${ }^{1}$, Rodrigo Erthal Wilson ${ }^{1,3}$, Vanussa da Silva Charles ${ }^{1}$, \\ Gil de Carvalho ${ }^{1}$ and Joaquim Teixeira de Assis ${ }^{1}$ \\ 1. Politechnical Institute of Rio de Janeiro State University, Nova Friburgo 28625-570, Brazil \\ 2. Estacio de Sá University, Nova Friburgo 28611-135, Brazil \\ 3. Federal Fluminense University, Itaocara 28470-000, Brazil
}

\begin{abstract}
This paper presents a method for determining the percentage of coarse aggregate in concrete specimens by image processing. The test pieces were produced with the aim of obtaining images of their cross sections through a scanner table. In order to increase the contrast between mortar and coarse aggregate the sliced surfaces were treated with the phenolphthale in solution. The images obtained in the scanner were processed in a program developed with MATLAB (matrix laboratory). The average coarse aggregate in each section and the mean of coarse aggregate per test body were calculated. With the results, it was revealed that the method returned satisfying results when compared to the original trace of the concrete.
\end{abstract}

Key words: Concrete, image processing, characterization of concrete.

\section{Introduction}

The concrete is a composite material composed of a ceramic matrix (cement) and a dispersed phase ceramic (fine and coarse aggregates). It may also contain additions and additives in order to improve or modify its basic properties [1]. The portland cement concrete, comes from the mixture of fine aggregate, usually sand washed, the coarse aggregate, usually crushed rock and the solid body connecting parts aggregate to each other, which is portland cement and water [2].

Portland cement is a fine powder with binders properties, which hardens under water action. Once hardened, even if it is subjected again to the action of water, does not decompose further.

It results from a product called clinker, obtained by cooking until the incipient fusion a mixture of limestone and clay dosed and homogenised

\footnotetext{
*Corresponding author: Jose Renato de Castro Pessoa, Professor, research fields: concrete technology and materials characterization. E-mail: jpessoa@iprj.uerj.br.
}

conveniently, so that all the calcareous combines with the clay compounds, without any amount of damaging free lime, after baking. After firing, a small addition of calcium sulfate is made so that the content of $\mathrm{SO}_{3}$ does not exceed $3.0 \%$ in order to adjust the starting time of the reactions of the binder with the water [3].

Approximately $3 / 4$ of the volume of concrete is occupied for aggregate. They not only limit the strength of concrete, as well as their properties significantly affect the durability and structural performance of concrete. From economic point of view, it is advantageous to produce mixtures with higher content of aggregate and the least amount possible of cement. This relationship needs to be balanced with the desired properties of concrete both in states, fresh and hardened [2].

The most important aggregate classification is that which divides them according to their size, this because of the very different behavior of both types when applied in the concrete. This classification 
subdivided them into fine aggregate and coarse aggregate [3]. The fine aggregate, according to NBR7211 (1983) [4] is defined as sand of natural origin resulting of crushing stable rock, or a mixture of both materials, in which the grains pass through ABNT (Brazilian national standards organization) $4.8 \mathrm{~mm}$ sieve and are retained on ABNT $0.075 \mathrm{~mm}$ sieve. The coarse aggregate, according to NBR 7211 (1983) [4] is defined as gravel or crushed rock from stable rock, or a mixture of both, in which the grains pass through a square mesh sieve with a nominal aperture of $152 \mathrm{~mm}$ and are retained on the $4.8 \mathrm{~mm}$ sieve.

The junction between fine and coarse aggregates on the composition of concrete has the function of getting a good dense packing in the final product. The final particles of sand fill the voids between the aggregates causing dense packing as desired. The whole set of aggregates should be added so that the amount of paste (cement and water) is sufficient to cover it compeletely, otherwise the cement binding will not occur [2].

The phenolphthalein is an acid indicator, i.e., has ionizable hydrogen in its structure. When the medium is acid ( $\mathrm{pH}$ less than 7), the indicator molecule is "forced" to keep their hydrogens due to the common ion effect, when the medium is basic ( $\mathrm{pH}$ greater than 7), the hydrogens of the indicator are strongly attracted by hydroxyl groups to form water, and in this case the anions of indicator are released that would color the medium [5]. The phenolphthalein has a pink color to the concrete in a range of $\mathrm{pH}$ of 8.0 to 10.0 [6].

The objective of this work is to calculate the percentage of coarse aggregate present in the concrete test bodies, using images obtained from a commercial scanner, using phenolphthalein on the surfaces of the test pieces to accentuate the contrast between the paste and the coarse aggregate in acquired images.

\section{Methodology and Experimental Procedure}

For this study three samples of $20 \mathrm{~cm}$ height and 10 cm in diameter were made, according to NBR 5738 (1994) [7]. The tests bodies were molded in "Laboratory of Physical Testing” of UERJ-IPRJ, Nova Friburgo. We used a composition for concrete with an expected resistance of $20 \mathrm{MPa}$ after 28 days. Table 1 shows the amount of components used to $1 \mathrm{~m}^{3}$ of concrete.

Damp curing of the test pieces was performed in water tank according to ABNT NBR 9479 (1994) [8], started immediately after concreting them. After 28 days the samples were sliced with cuts every $5 \mathrm{~cm}$, thus obtaining 4 slices per test body. Each slice of samples received an identification with the growing number from 1 to 4 , where the slice number 1 refers to the upper slice and slice number 4 refers lower slice. Fig. 1 shows the test bodies sliced and identified.

After, the phenolphthalein solution application was made on the inner surfaces of the test pieces. Fig. 2 shows the surface of one of the slices of the test body after application of phenolphthalein solution.

After treatment of the surfaces with the solution of phenolphthalein and complete drying of the same, we obtained images of the internal surfaces of the test body using a commercial scanner HP DESKJET

Table 1 Composition used to $1 \mathbf{~ m}^{3}$ of concrete.

\begin{tabular}{ll}
\hline Components & Mass for $\left(\mathrm{m}^{3}\right)$ \\
\hline Cement CP II E 32 & $350 \mathrm{~kg}$ \\
Sand & $830 \mathrm{~kg}$ \\
Gravel 0 & $100 \mathrm{~kg}$ \\
Gravel 1 & $830 \mathrm{~kg}$ \\
Water & $223 \mathrm{~L}$ \\
\hline
\end{tabular}

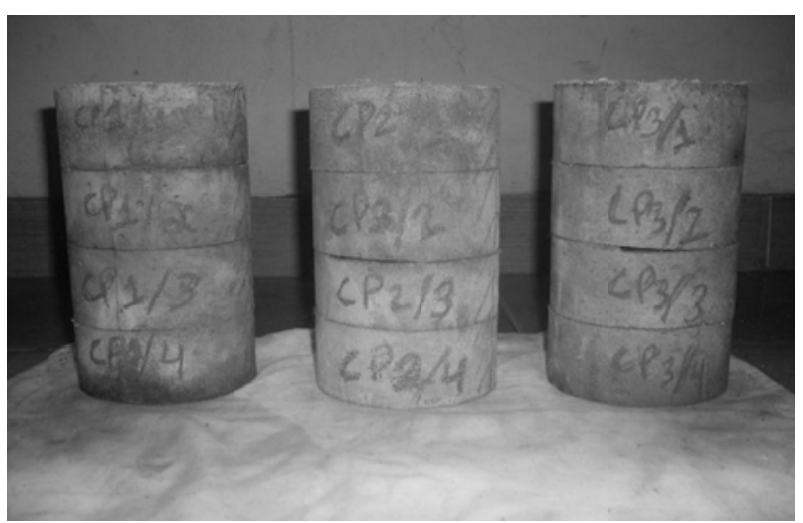

Fig. 1 Test bodies sliced and identified. 

Digital Images Obtained with a Commercial Scanner

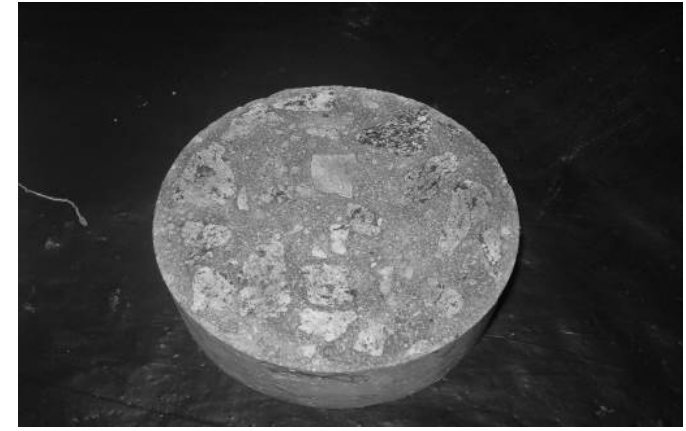

Fig. 2 Surface of one of the slices of the test body after application of phenolphthalein solution.

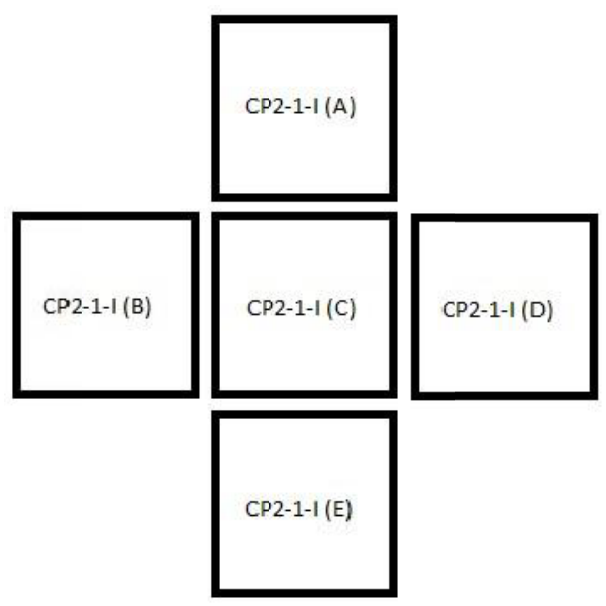

Fig. 3 Identification of the images on each surface of the test pieces. (CP2-1-I(A) : image A of the bottom surface of the first slice of the test body 2 ).

F4180 with a resolution of 2,400 dpi. The external surfaces of the test body were excluded from the analysis. For each surface five images were obtained. The nomenclature used to identify each image is shown in Fig. 3.

A program in MATLAB was used, to read the images and process them in order to determine the percentage of coarse aggregate present in the samples. The resulting image is binarized image where the number of black pixels are interpreted as coarse aggregate. Fig. 4 shows the processing steps for one image.

We analyzed 30 images in each test body, totalizing 90 images in all of samples.

\section{Results and Discussion}

Table 2 shows the results for the average amount of coarse aggregate per test body after the analysis of 90 images.

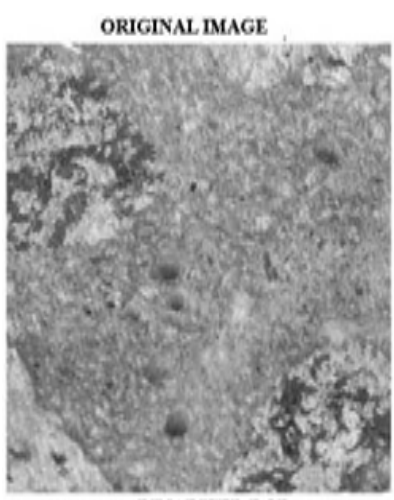

BINARIZED RGB

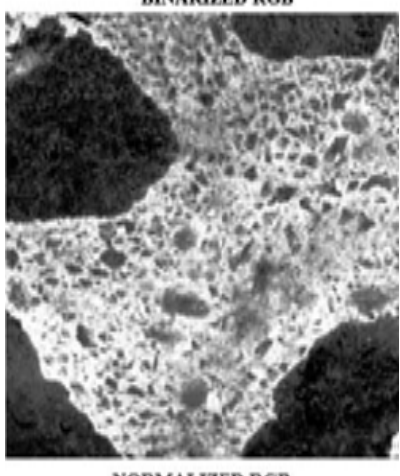

NORMALIZED RGB

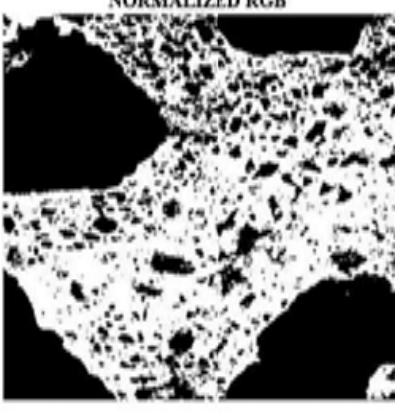

BYNARY CLOSING + OPENING
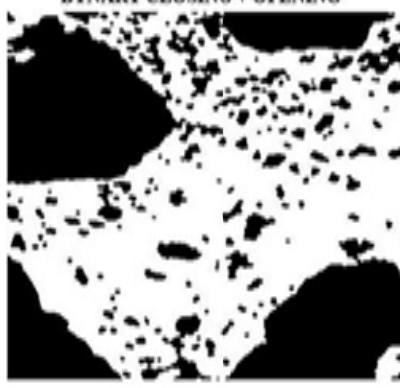

REMOVING NOISE
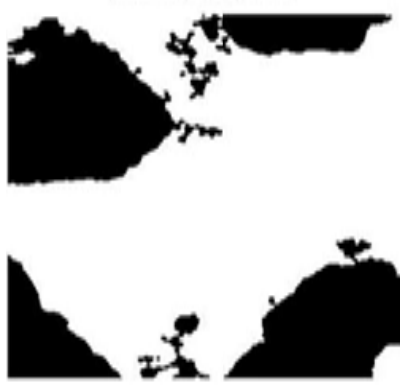

Fig. 4 Stages of processing an image obtained. 
Digital Images Obtained with a Commercial Scanner

Table 2 Average amount of coarse aggregate by test body.

\begin{tabular}{lll}
\hline $\begin{array}{l}\text { Test } \\
\text { body }\end{array}$ & $\begin{array}{l}\text { Average of coarse } \\
\text { aggregate by body (\%) }\end{array}$ & $\begin{array}{l}\text { Total average of coarse } \\
\text { aggregate in concrete (\%) }\end{array}$ \\
\hline 1 & 38.8 & \\
2 & 38.5 & 37.87 \\
3 & 36.3 & \\
\hline
\end{tabular}

For comparison, it was necessary to get the real percentage of coarse aggregate present in the concrete samples according to the data shown in Table 1 . The real value of coarse aggregate according Table data is $32.67 \%$.

With the results we observed that the method of image processing used to determine the percentage of coarse aggregate returned results with positive difference of approximately $16 \%$.

The likely cause of this difference is the "threshold" cutting binarization of the program developed in MATLAB, which can making some noise to be read as being aggregate.

\section{Conclusions}

We conclude that the method has reached a satisfactory result when compared with the effectively amount of coarse aggregate in the mixture, some adjustments must be made in developed program.

The use of commercial scanner to obtain images of concrete is presented as a non-destructive method acceptable by their results and especially for its low cost and ease of operation.

\section{Acknowledgments}

The authors acknowledge the support given by the Carlos Chagas Filho Foundation for Research Support of the State of Rio de Janeiro FAPERJ (Fundação Carlos Chagas Filho de Amparo à Pesquisa do Estado do Rio de Janeiro), the Coordination of Improvement of Superior Level Personal CAPES (Coordenação de Aperfeiçoamento de Pessoal de Nível Superior) and National Council for Scientific and Technological Development CNPQ (Conselho Nacional de Desenvolvimento Científico e Tecnológico).

\section{References}

[1] Callister, W. D. 2008. Materials Science and Engineering. Rio de Janeiro: LTC.

[2] Neville, A. M., and Brooks, J. J. 2013. Concrete Technology. São Paulo: Bookman.

[3] Petrucci, E. G. R. 1998. Portland Cement Concrete. Porto Alegre: Globo.

[4] Brazilian Association of Technical Standards-NBR 7211. 1983. Aggregate for Concrete. Rio de Janeiro, ABNT.

[5] Maia, D. J., and Bianchi, J. C. A. 2007. Química Geral. São Paulo: Pearson.

[6] Peterson, K. 2001. "Air Void Analysis of Hardened Concrete by Scanner.” Master Theses, Michigan Technological University.

[7] Brazilian Association of Technical Standards-NBR 5738. 1994. Molding and Curing of Cylindrical or Prismatic Concrete Test Pieces. Rio de Janeiro.

[8] Brazilian Association of Technical Standards-NBR 9479. 1994. Humid Chambers and Tanks for Curing Body-of-Proof Mortar and Concrete. Rio de Janeiro: ABNT. 